\title{
Clinical and structural outcome 22 years after acromioplasty without tendon repair in patients with subacromial pain and cuff tears
}

Mats Ranebo, Hanna Björnsson Hallgren, Rolf Norlin and Lars Adolfsson

The self-archived postprint version of this journal article is available at Linköping University Institutional Repository (DiVA):

http:/ / urn.kb.se/ resolve?urn=urn:nbn:se:liu:diva- 140975

N.B.: When citing this work, cite the original publication.

Ranebo, M., Björnsson Hallgren, H., Norlin, R., Adolfsson, L., (2017), Clinical and structural outcome 22 years after acromioplasty without tendon repair in patients with subacromial pain and cuff tears, J ournal of shoulder and elbow surgery, 26(7), 1262-1270. https:// doi.org/10.1016/j.jse.2016.11.012

Original publication available at:

https:// doi.org/ 10.1016/j.jse.2016.11.012

Copyright: Elsevier

http:// www.elsevier.com/

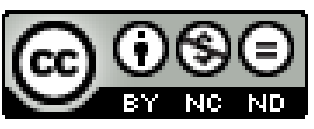


22-year follow-up of unrepaired cuff tears

Title

\section{Clinical and structural outcome 22 years after acromioplasty} without tendon repair in patients with subacromial pain and cuff tears.

Short-form title: 22-year follow-up of unrepaired cuff tears

Mats C. Ranebo, MD ${ }^{\mathrm{a}, \mathrm{c}}$, Hanna C. Björnsson Hallgren, $\mathrm{MD}, \mathrm{PhD}^{\mathrm{b}}$, Rolf Norlin, $\mathrm{MD}, \mathrm{PhD}^{\mathrm{c}}$, Lars E. Adolfsson, $\mathrm{MD}, \mathrm{PhD}^{\mathrm{b}, \mathrm{c}}$.

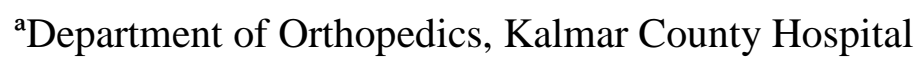

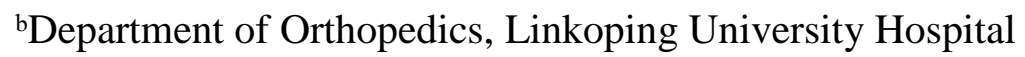

cThe Faculty of Medicine and Health Sciences, Linköping University.

Corresponding author: Mats Ranebo, M.D, Department of Orthopaedics, Kalmar County Hospital, 39185 Kalmar, Sweden

E-mail: mats.ranebo@ltkalmar.se

Disclaimer: None

Ethical Committee approval: Regional Ethics Committee Linkoping, Dnr 2013/330-31.

\section{Acknowledgements}

The authors would like to thank Lars Brudin for statistical advice and Lena Törnqvist for help with radiological analysis. 


\title{
22-year follow-up of unrepaired cuff tears
}

\begin{abstract}
Background: Long-term results regarding tear progression, arthropathy and clinical scores of unrepaired rotator cuff tears are largely unknown. The purposes of this study were to investigate if the condition of the glenohumeral joint and rotator cuff had deteriorated at a minimum of 20 years after an acromioplasty without cuff repair and to assess the clinical results.
\end{abstract}

Methods: Retrospective analysis of a consecutive series of patients treated between 1989 and 1993 with acromioplasty without cuff repair due to subacromial pain and cuff tear. At followup results of x-ray, ultrasound (US) and clinical scores were recorded.

Results: At a mean of 22 years (range 21-25), 69 patients were available for follow-up with WORC, Constant score (CM), x-ray and US. Mean age at operation was 49 years (range 1969). There were 45 partial-thickness tears (PTT) and 24 full-thickness tears (FTT). In patients with FTT 74\% (17/23 with X-ray) had developed cuff tear arthropathy (CTA, Hamada $\geq 2)$ and 87\% (20/23 with US) had progressed in tear size. Mean relative CM in patients with FTT and CTA was 62 (SD 27), mean WORC 58\% (SD 26). In PTT-patients 7\% (3/43 with x-ray) had developed CTA and 42\% (16/38 with US) tear progression. With PTT at follow-up mean relative CM was 101 (SD 22), mean WORC 81\% (SD 20).

Conclusions: Following an acromioplasty most unrepaired full thickness tears will, in longterm, increase in size and be accompanied by CTA changes. The majority of partial thickness tears remain unchanged; CTA is rare and clinical scores generally good.

Level of evidence: Level III, Retrospective Comparative Study, Treatment study.

Keywords: Shoulder; rotator cuff; long-term; tear progression; cuff tear arthropathy; acromioplasty; natural history. 


\section{2-year follow-up of unrepaired cuff tears}

\section{Introduction}

The optimal treatment for rotator cuff tears is still controversial ${ }^{7,45}$. Published results of rotator cuff repair are generally good ${ }^{12,32}$, but the retear rate is reported to be between $11 \%$ and $94 \%{ }^{14,32}$ and the rehabilitation period is sometimes extensive ${ }^{29}$. Randomized trials on rotator cuff repair compared to acromioplasty or only physiotherapy have not yet shown a clinically relevant difference in outcome in the short- to medium term ${ }^{31,37}$. Potential drawbacks of not repairing a cuff tear are tear progression ${ }^{27,33,41}$, functional deterioration and development of cuff tear arthropathy ${ }^{21,38}$. The structural deterioration is likely to be a slow process over several years and the clinical significance is largely unknown. The follow-up period in studies on conservative treatment or subacromial decompression without repair is generally of short- to medium term ${ }^{10,11,15,26,33,41,50}$ and long-term studies that include structural changes of the shoulder joint are lacking ${ }^{22}$.

The overall aim of this study was to investigate if the condition of the glenohumeral joint and rotator cuff had deteriorated at a minimum of 21 years after an acromioplasty, without cuff repair, in patients with partial and full-thickness rotator cuff tears. The two main outcomes were tear progression and cuff tear arthropathy. A secondary aim was to examine the clinical results. 


\section{2-year follow-up of unrepaired cuff tears}

\section{Materials and methods}

The study was approved by the local ethics committee at the University Hospital of Linköping in Sweden (Dnr 2013/330-31).

A consecutive series of 111 patients with an intraoperative finding of a rotator cuff tear was identified through all surgical protocols from patients having undergone an arthroscopic subacromial decompression at Linköping University Hospital between 1989 and 1993. During this period it was believed that the majority of patients with subacromial pain had a good outcome of an arthroscopic subacromial decompression (ASD) only. Therefore all patients that needed surgery were initially treated with only ASD regardless of the cuff condition. At follow-up 21 patients were dead, 11 were medically too ill to be contacted and 1 patient could not be located. The remaining 78 patients were contacted and 69 accepted participation, resulting in a follow-up rate of $88 \%$ for eligible patients. A flow-chart of the inclusion process is presented in fig.1. Each patient gave written informed consent. Medical charts were retrospectively reviewed. All included patients had preoperatively suffered from shoulder pain for more than six months, and had been diagnosed with subacromial pain or impingement syndrome by an orthopedic surgeon. Preoperative standard x-rays, with frontal and lateral projections were without signs of osteoarthritis or cuff tear arthropathy. Before surgery all patients had been treated with a period of physiotherapy and at least one subacromial corticosteroid injection.

\section{Surgical technique}

The operations were performed in the lateral decubitus position by one of four experienced senior shoulder surgeons. A standard posterior portal was used for the arthroscope and a lateral portal for instruments. Routinely the glenohumeral joint was examined according to a specially designed protocol. All findings were documented in the protocol where the 


\section{2-year follow-up of unrepaired cuff tears}

alternatives for integrity of each rotator cuff tendon were: intact, articular side partial tear, bursal side partial tear or full-thickness tear. There was no requirement of tear size measurements but the location of a tear was indicated in a drawing. Subacromial bursal resection and an acromioplasty was performed including release of the coracoacromial ligament. The anterolateral undersurface of the acromion was resected to a flat contour with a slight upward slope towards the insertion of the CA ligament. The resection typically aimed at creating a distance equaling a distance twice the width of the $6 \mathrm{~mm}$ shaver blade between the supraspinatus tendon and the acromion. Any osteophytes surrounding the undersurface of the acromioclavicular joint were resected. No rotator cuff tears identified during arthroscopy were repaired or trimmed. The biceps tendon was not subjected to any kind of measure in any of the operated patients. Postoperatively the patients wore a sling for comfort. Postoperative rehabilitation was guided by a physiotherapist.

\section{Clinical assessment at follow-up}

The follow-up assessment was performed at 21-25 years after the index operation by two examiners (M.R and H.BH) not involved in the operations. Every patient filled out the Western Ontario Rotator Cuff index (WORC) ${ }^{28}$ and patients who could attend a clinical examination were also assessed by the Constant-Murley score ${ }^{5}$. Abduction strength for this score was measured with a validated digital myometer (McMesin Myometer ${ }^{\circledR}$, Slinfold, West Sussex, UK) ${ }^{24}$. The absolute and the age- and gender adjusted relative Constant-Murley scores were calculated according to the original publication ${ }^{5}$. The 2 questions relating to pain in the WORC-score were analyzed separately as the millimeter measurement on the $100 \mathrm{~mm}$ visual analogue scale for each question. The pain-questions in WORC relate to the last week. Question number 1 reads: "How much sharp pain do you experience in your shoulder?". Question number 2 reads: "How much constant nagging pain do you experience in your shoulder?”. For both questions a division into four categories were done according to Jensen 


\section{2-year follow-up of unrepaired cuff tears}

et $\mathrm{al}^{23}$ : no pain 0-4 mm, mild pain 5-44 mm, moderate pain 45-74 mm and severe pain $75-100$ $\mathrm{mm}$.

\section{Ultrasonography}

Ultrasonography was performed bilaterally with a BK Medical Flex Focus 500 (BK Medical, Peabody MA, USA) with a variable high frequency linear array transducer (6-18 MHz). All examinations were performed according to a previously described protocol by one of the authors (H.BH),, an orthopedic surgeon with extensive ultrasound experience ${ }^{3}$. Tendon lesions were classified as partial-thickness tears if there was a hypoechoic defect in both transverse and longitudinal planes on the articular or bursal side. A full-thickness tear was diagnosed when there was a hypoechoic defect in the whole thickness of the tendon visualized in two planes. Tear progression was defined as progression from a partial thickness tear at the primary operation to a full-thickness tear at follow-up, or as progression from a full-thickness tear at the primary operation to a full-thickness tear in an additional tendon at follow-up. A partial tear in an additional tendon at follow-up was not considered tear progression.

\section{Radiographic examination and analysis at follow-up}

Bilateral standard frontal and lateral projections were taken. The X-ray images were independently classified by members of the study group (M.R, H.BH, L.A) and by an independent radiologist according to the Hamada classification ${ }^{18,19}$. The acromiohumeral distance was measured in the anteroposterior projection as the shortest distance from the most superior part of the subchondral bone of the humeral head to the dense cortical bone of the undersurface of the acromion with the measurement tool in the digital workstation PACS (Sectra, Linkoping, Sweden). Controversies on classification grade were resolved by consensus discussion.

\section{Statistics}




\section{2-year follow-up of unrepaired cuff tears}

The sample size represents the available patients to be examined from the predefined period. Descriptive statistics with mean values and standard deviation as well as median values and quartile range were used for continuous data. For categorical data frequencies were calculated as percentage of patients with available data. When comparing groups with categorical data Fischer's exact test was used and for numerical data the Mann-Whitney U-test. Kappa statistics were used for interobserver reliability regarding Hamada classification of radiographic images. To assess risk factors for tear progression and arthropathy multivariate logistic regression with stepwise forward and backward selection methods were used. The statistical software package utilized was Statistica ${ }^{\circledR} 12.0$ (StatSoft, Tulsa, OK, USA) and the statistical significance was set at $\mathrm{p}<.05$. 


\section{2-year follow-up of unrepaired cuff tears}

\section{Results}

Sixty-nine operated patients (69 shoulders) were available for follow-up at a mean of 22 years (range 21-25 years) after the index operation. Mean age at surgery was 49 years (range 19-69) and there were 45 partial tears and 24 full-thickness tears. The demographic data, operative findings of tendon status and available data at follow-up are shown in table I. Sixty-one patients were able to visit the clinic for complete follow-up, the remaining 8 patients completed WORC and 5 of these also underwent x-ray examination (table I). Mean age at follow-up was 72 years (range 43-92 years) for the whole cohort. For patients with partial tears at index operation the mean age was 69 years (range 43-88) and for patients with fullthickness tears 77 years (range 66-92). The patients with partial tears were significantly younger than those with full thickness tears $(\mathrm{p}=0.003)$.

\section{Tear progression}

The total rate of tear progression in the full-thickness group was $87 \%$. In patients with only a full-thickness tear in supraspinatus at the index operation 16\% had no tear progression, 32\% had developed a tear in infraspinatus as well and 53\% had tears in supraspinatus, infraspinatus and subscapularis (table II). The 4 patients with supra- and infraspinatus tears at the operation all had progression to a full-thickness tear in the subscapularis as well.

The total rate of tear progression in the partial tear group was $42 \%$. In this group $24 \%$ had developed a full-thickness tear in only supraspinatus, $16 \%$ full-thickness tears involving both supra- and infraspinatus and 1 patient also had a tear in subscapularis (table II). The difference in frequency of tear progression between partial and full-thickness tears was statistically significant $(\mathrm{p}<0.001)$.

\section{Radiographic outcome}




\section{2-year follow-up of unrepaired cuff tears}

Thirty percent of the whole cohort had developed signs of cuff tear arthropathy corresponding to Hamada 2 or more at follow-up. In the full-thickness group 74\% had Hamada 2 or more (table III). Thirty percent in this group had Hamada 4b, indicating severe arthropathy. In the partial thickness group 7\% (3 patients) had Hamada 2 or more. One patient with an initial partial tear had Hamada 4b changes. This patient had not sustained a trauma during the follow-up period but his ultrasound examination showed full-thickness tears in supra- and infraspinatus. Signs of cuff tear arthropathy was significantly more common in patients having full-thickness tears at the index operation compared to patients having partial tears $(\mathrm{p}<0.001)$. Two patients had an $\mathrm{x}$-ray at follow-up indicating osteoarthritis, i.e. a decreased joint space and inferior osteophytes on the humeral head but no decrease of the acromiohumeral distance and no acetabulization and their x-rays were interpreted as Hamada 1. The Kappa values for Hamada classification for 3 assessors were 0.83, 0.76 and 0.75 .

\section{Clinical results}

Clinical scores in relation to tear type and radiographic outcome at follow-up are displayed in table IV. The group with a full-thickness tear and Hamada $4 \mathrm{~b}$ at follow-up had the lowest values in Constant score (mean relative score 51, SD 20) and WORC (mean WORC 48\%, SD 19). Patients with Hamada grade 1 despite a full-thickness tear at follow-up had a mean relative Constant score of 88 (SD 38) and a mean WORC of 72\% (SD 26). Patients with a partial-thickness tear and a normal x-ray at follow-up had a mean relative Constant score of 101 (SD 22) and a mean WORC of 81\% (SD 20).

Patients with a partial tear at primary operation had a mean WORC of 77\% (SD 22) and a mean relative CM of 92 (SD 32) at follow-up, with $72 \%$ of the patients having a relative CM of 80 or more. Patients with a full-thickness tear at index operation had a mean WORC of $64 \%$ (SD 27) and a mean relative CM of 72 (SD 33) at follow-up, with 43\% having a relative 


\section{2-year follow-up of unrepaired cuff tears}

CM of 80 or more. There was a significant difference in relative Constant score $(p=0.027)$ and WORC ( $\mathrm{p}=0.030)$ between patients with full-thickness tears and partial tears at index operation.

The number of patients with and without pain as well as the mean VAS-scores for both sharp pain and constant nagging pain is presented in table IV according to tendon status and radiographic status at follow-up. Approximately half of the patients with either partial or fullthickness tears with normal x-ray had some kind of pain, and the majority of those had mild pain. Patients with full-thickness tears and Hamada arthropathy grade 2 or more had moderate to severe pain in $30-35 \%$ of the cases (table IV).

\section{Reoperations}

The total rate of reoperations for all kinds of shoulder operations in the index shoulder was $22 \%$ and the corresponding reoperation rates for partial and full-thickness tears were $18 \%$ and 29\%. The number and types of reoperations are displayed in table V. Two patients with fullthickness tears underwent later attempts at cuff repair, none of these repairs were intact at follow-up. Three patients with irreparable cuff tears were operated with a Dacron patch to cover the defect, but at follow-up the patch was not intact in any of these patients.

\section{Additional trauma after index operation}

A total of nine patients had sustained a trauma during the follow-up period. Four patients with a full-thickness tear at index operation had sustained a trauma and 3 of those had a larger tear at follow-up. Five patients with a partial-thickness tear had sustained a trauma and in 3 of those the tear had progressed to a full-thickness lesion.

\section{Risk factor analysis for arthropathy and tear progression}




\section{2-year follow-up of unrepaired cuff tears}

We analyzed age, gender, reoperation, tendon status at index operation, biceps status at index operation, traumatic tear and manual labor as factors predicting cuff tear arthropathy. In a logistic regression model with Hamada grade 2 or more as dependent variable only having a full-thickness tear at index operation came out as a significant variable $(\mathrm{p}<0.001)$ with odds ratio 37.8 (95\% CI: 8.2-175.0). The same factors (except biceps status) were also analyzed for predicting tear progression and both age $(\mathrm{p}=0.043)$ with odds ratio 1.07 (95\% CI: 1.00-1.15) and having a full-thickness tear ( $\mathrm{p}=0.016)$ with odds ratio 6.09 (95\% CI: 1.41-26.29) were significant factors. Since all patients with biceps pathology at index operation had tear progression this factor was not possible to include in a logistic regression model, but when analyzed separately with tear progression in a Fischer's test the distribution was not significantly different than expected $(\mathrm{p}=0.072)$. 


\section{2-year follow-up of unrepaired cuff tears}

\section{Discussion}

In this long-term follow-up study of patients treated with acromioplasty without cuff repair we found a high frequency of tear progression and cuff tear arthropathy, defined as Hamada 2 or more, after 21-25 years in patients with a full-thickness rotator cuff tear. The radiological findings were reflected by lower shoulder scores in this group and patients without radiographic signs of cuff tear arthropathy despite the presence of a full thickness tear at follow-up scored better. In patients with partial-thickness tears, a majority had no arthropathy and high scores.

Tear progression is important since progression may make a tear irreparable and an increase in tear size has been found correlating with increased symptoms ${ }^{36}$. It is known from recent longitudinal studies with 2 to 5 year follow-up with ultrasound or MRI that both asymptomatic and symptomatic tears often progress, for full-thickness tears in around 49-61\% of the cases $27,33,36,41$. Our findings of tear progression rates of $87 \%$ for full-thickness tears and $42 \%$ for partial tears more than 20 years after initial diagnosis is in accordance with these studies, albeit perhaps lower than expected considering our long follow-up period. Ultrasound is a well-established method with a high degree of accuracy in detecting full-thickness tears but lower accuracy for partial tears ${ }^{20,34,46}$. Consequently we did not consider an ultrasound diagnosis of a partial tear in an additional tendon at follow-up as tear progression. This more conservative definition could have led to a slight underestimation of progression rates.

Forty-two percent of patients with initial partial tears had a full-thickness tear at a mean age of 69 years, which is higher than the expected $10-25 \%$ among people in their $60 \mathrm{~s}^{35,48}$. Considering that our cohort were selected on the basis of having a rotator cuff problem this is however not surprising. 


\section{2-year follow-up of unrepaired cuff tears}

The Hamada classification is frequently used and has performed well in comparison with other cuff tear arthropathy classifications ${ }^{25}$. The Kappa values regarding Hamada classification for 3 assessors indicated good to excellent agreement according to interpretation guidelines ${ }^{44}$.

The arthropathy stage of a patient is an important consideration in treatment decision-making for symptomatic patients. A decreased acromiohumeral interval (as in Hamada 2) has been shown to be associated with massive tears with fatty infiltration of supra- and infraspinatus and is considered an important risk-factor for repair failure ${ }^{9,40}$. In patients with an irreparable cuff tear the decision-making for more advanced surgical alternatives such as tendon transfer or reverse arthroplasty relies to a certain extent on the x-ray images ${ }^{8,47}$. In this study all patients had been treated with an acromioplasty, a procedure aimed at modifying the undersurface of the acromion potentially making the distinction between Hamada 2 and 3 more difficult. Theoretically an extensive acromioplasty could also conceal a true Hamada 2 but in a 20 year perspective this is unlikely to have a substantial influence. It cannot be ruled out that the acromioplasty might have had a negative impact on the degenerative changes in patients with a full-thickness tear. The numbers in each category should however be interpreted with caution.

Studies with long-term results of more than 10 years after acromioplasty in patients with cuff tears are few and the present study is to our knowledge the only one reporting 22-year results with structural outcome included ${ }^{22,39}$. Jaeger et $\mathrm{al}^{22}$ recently published 20-year results of Constant score and reoperations after acromioplasty in 95 patients with both intact and torn rotator cuffs, but the study includes no analysis of joint or rotator cuff status and the follow-up rate was only $57.2 \%$. Their outcome, defined as a measure of successful treatment, was a combined endpoint of relative Constant score above 70 and no reoperation. Ninety-one percent of the patients with a partial tear and $71 \%$ with a full-thickness tear had a successful 


\section{2-year follow-up of unrepaired cuff tears}

result. With this definition we found that $64 \%$ of the partial tear patients and $39 \%$ of the fullthickness tear patients in our cohort had a successful outcome. The difference can in part be explained by a much higher follow-up rate in our study (88\% vs 57.2\%) and perhaps in different indications for reoperation.

In the present study 32 patients from the initial cohort of 111 patients were either dead or too ill to be examined. Since some of the risk factors for development of a degenerative cuff tear are the same as for cardiovascular disease and cancer ${ }^{1,6,17,45}$ one may suspect that the rates of tear progression and cuff tear arthropathy would have been high in these patients if they had been examined. The finding that 21 out of 111 patients (19\%), with a mean age at surgery of 49 years, had died is something that might warrant further investigation.

The WORC and CM scores may appear high considering the structural deficits, but this finding is in line with earlier studies ${ }^{22,39}$. In a study on reversed subacromial decompression and biceps tenotomy in patients with massive tears, Scheibel et al found similar discrepancy between structural deterioration and preserved scores ${ }^{43}$. Zingg et al also reported the same phenomenon in a 4-year follow-up study on conservatively treated massive tears, where a satisfactory shoulder function was maintained despite progression of degenerative joint changes $^{49}$. The perhaps most important aspect of patient reported outcome in shoulder care is pain. In this study around half of patients with an x-ray without arthropathy had a pain-free shoulder, regardless of the presence of a partial or a full-thickness tear. Patients with pain in these two groups had a mild pain in most cases but $12-14 \%$ had a moderate to severe pain. With a full-thickness tear and Hamada arthropathy grade 2 or more around one third of the patients had moderate to severe pain. In patients with full-thickness tears the long-term risk of ending-up with a moderately to severely painful shoulder seems not to be negligible. 


\section{2-year follow-up of unrepaired cuff tears}

Some patients with full-thickness tears of only supraspinatus in our study were able to maintain a reasonable shoulder function and an x-ray without arthropathy despite having the problem for more than 20 years. An intact rotator cable ${ }^{4}$ and a preserved antero-posterior force couple could be possible explanations.

This study cannot answer the question whether a tendon repair would have resulted in better outcome. Prospective randomized studies with 2-5 year results have not shown any clinically significant difference between repair and non-repair strategies ${ }^{31,37}$. In a prospective study on repair of full-thickness tears Galatz et al at ten-year follow up reported 91\% good or excellent results, defined as a relative Constant score of 80 or more ${ }^{13}$. In our cohort of 24 patients with unrepaired full-thickness tears the corresponding rate of patients having a relative CM of 80 or more was $43 \%$. These are small cohorts with a large difference in follow-up time and differences in results should therefore be interpreted with caution. Our results on tear progression and cuff tear arthropathy appear to emphasize the need for careful selection of patients considered for repair.

This study has limitations. We only have preoperative radiographs and perioperative tendon status for comparison and no preoperative clinical scores. We also did not have access to intraoperative measurements of tear size, which could have made the estimate of true tear progression more accurate. The risk of misclassifying a full-thickness tear as a partial tear at arthroscopy is probably low but classifying the depth of a partial tear at arthroscopy is a known problem ${ }^{30}$. The Hamada classification rely on measurement of acromiohumeral interval on plain radiographs for distinction between normal and type 2. This measurement on plain radiographs has been noted to have some observer variability in one study ${ }^{2}$ but acceptable variability in another study ${ }^{16}$ and appears acceptable when compared to measurement with MR-arthrography ${ }^{42}$. We tried to overcome the risk of potential observer bias by having three independent assessors and found an acceptable agreement. 


\section{2-year follow-up of unrepaired cuff tears}

\section{Conclusion}

At a mean of 22 years after an acromioplasty without cuff repair, patients with full-thickness tears at the index operation were found to have a high frequency of tear progression and cuff tear arthropathy. The clinical results were inferior in patients with arthropathy, but otherwise appeared reasonable despite the presence of a full-thickness tear. In patients with partial tears a majority had no tear progression and a very low rate of cuff tear arthropathy. These results indicate that rotator cuff tear is a potentially progressive disease and that individuals with fullthickness tears appear to have a higher risk for clinical and structural deterioration. 


\section{2-year follow-up of unrepaired cuff tears}

\section{References}

1. Abboud JA, Kim JS. The effect of hypercholesterolemia on rotator cuff disease. Clin Orthop Rel Res 2010;468(6):1493-7. doi: 10.1007/s11999-009-1151-9.

2. Bernhardt GA, Glehr M, Zacherl M, Wurnig C, Gruber G. Observer variability in the assessment of the acromiohumeral interval using anteroposterior shoulder radiographs. Europ J Orthop Surg \& Traumatol 2013;23(2):185-90. doi: 10.1007/s00590-012-0942-y.

3. Blinded for review purposes.Bjornsson HC, Norlin R, Johansson K, Adolfsson LE. The influence of age, delay of repair, and tendon involvement in acute rotator cuff tears: structural and clinical outcomes after repair of 42 shoulders. Acta orthopaedica 2011;82(2):187-92. doi: 10.3109/17453674.2011.566144.

4. Burkhart SS, Esch JC, Jolson RS. The rotator crescent and rotator cable: an anatomic description of the shoulder's "suspension bridge". Arthroscopy 1993;9(6):611-6.

5. Constant CR, Murley AH. A clinical method of functional assessment of the shoulder. Clin Orthop Relat Res 1987(214):160-4.

6. Djerbi I, Chammas M, Mirous MP, Lazerges C, Coulet B. Impact of cardiovascular risk factor on the prevalence and severity of symptomatic full-thickness rotator cuff tears. Orthop Traumatol Surg Res 2015;101(6 Suppl):S269-73. doi: 10.1016/j.otsr.2015.06.011.

7. Dunn WR, Schackman BR, Walsh C, Lyman S, Jones EC, Warren RF, et al. Variation in orthopaedic surgeons' perceptions about the indications for rotator cuff surgery. J Bone Joint Surg Am 2005;87(9):1978-84. doi: 10.2106/jbjs.d.02944.

8. El-Azab HM, Rott O, Irlenbusch U. Long-Term Follow-up After Latissimus Dorsi Transfer for Irreparable Posterosuperior Rotator Cuff Tears. J Bone Joint Surg Am 2015;97(6):462-9. doi: 10.2106/jbjs.m.00235.

9. Ellman H, Hanker G, Bayer M. Repair of the rotator cuff. End-result study of factors influencing reconstruction. J Bone Joint Surg Am 1986;68(8):1136-44. 


\section{2-year follow-up of unrepaired cuff tears}

10. Ellman H. Arthroscopic subacromial decompression: analysis of one- to three-year results. Arthroscopy 1987;3(3):173-81.

11. Fucentese SF, von Roll AL, Pfirrmann CW, Gerber C, Jost B. Evolution of nonoperatively treated symptomatic isolated full-thickness supraspinatus tears. J Bone Joint Surg Am 2012;94(9):801-8. doi: 10.2106/jbjs.i.01286.

12. Fuchs B, Gilbart MK, Hodler J, Gerber C. Clinical and structural results of open repair of an isolated one-tendon tear of the rotator cuff. J Bone Joint Surg Am 2006;88(2):309-16. doi: 10.2106/jbjs.e.00117.

13. Galatz LM, Griggs S, Cameron BD, lannotti JP. Prospective longitudinal analysis of postoperative shoulder function : a ten-year follow-up study of full-thickness rotator cuff tears. J Bone Joint Surg Am 2001;83-A(7):1052-6.

14. Galatz LM, Ball CM, Teefey SA, Middleton WD, Yamaguchi K. The outcome and repair integrity of completely arthroscopically repaired large and massive rotator cuff tears. J Bone Joint Surg Am 2004;86-A(2):219-24.

15. Goldberg BA, Nowinski RJ, Matsen FA, 3rd. Outcome of nonoperative management of fullthickness rotator cuff tears. Clin Orthop Relat Res 2001(382):99-107.

16. Gruber G, Bernhardt GA, Clar H, Zacherl M, Glehr M, Wurnig C. Measurement of the acromiohumeral interval on standardized anteroposterior radiographs: a prospective study of observer variability. J Shoulder Elbow Surg 2010;19(1):10-3. doi: 10.1016/j.jse.2009.04.010.

17. Gumina S, Arceri V, Carbone S, Albino P, Passaretti D, Campagna V, et al. The association between arterial hypertension and rotator cuff tear: the influence on rotator cuff tear sizes. J Shoulder Elbow Surg 2013;22(2):229-32. doi: 10.1016/j.jse.2012.05.023.

18. Hamada K, Fukuda H, Mikasa M, Kobayashi Y. Roentgenographic findings in massive rotator cuff tears. A long-term observation. Clin Orthop Relat Res 1990(254):92-6. 


\section{2-year follow-up of unrepaired cuff tears}

19. Hamada K, Yamanaka K, Uchiyama Y, Mikasa T, Mikasa M. A radiographic classification of massive rotator cuff tear arthritis. Clin Orthop Relat Res 2011;469(9):2452-60. doi: 10.1007/s11999-011-18969.

20. Hinsley H, Nicholls A, Daines M, Wallace G, Arden N, Carr A. Classification of rotator cuff tendinopathy using high definition ultrasound. Muscles Ligaments Tendons J 2014;4(3):391-7. 21. Hsu HC, Luo ZP, Stone JJ, Huang TH, An KN. Correlation between rotator cuff tear and glenohumeral degeneration. Acta Orthop Scand 2003;74(1):89-94. doi: 10.1080/00016470310013725.

22. Jaeger M, Berndt T, Ruhmann O, Lerch S. Patients With Impingement Syndrome With and Without Rotator Cuff Tears Do Well 20 Years After Arthroscopic Subacromial Decompression. Arthroscopy 2015. doi: 10.1016/j.arthro.2015.08.026.

23. Jensen MP, Chen C, Brugger AM. Interpretation of visual analog scale ratings and change scores: a reanalysis of two clinical trials of postoperative pain. J Pain 2003;4(7):407-14.

24. Blinded for review purposes.Johansson KM, Adolfsson LE. Intraobserver and interobserver reliability for the strength test in the Constant-Murley shoulder assessment. J Shoulder Elbow Surg 2005;14(3):273-8. doi: 10.1016/j.jse.2004.08.001.

25. Kappe T, Cakir B, Reichel H, Elsharkawi M. Reliability of radiologic classification for cuff tear arthropathy. J Shoulder Elbow Surg 2011;20(4):543-7. doi: 10.1016/j.jse.2011.01.012.

26. Kartus J, Kartus C, Rostgard-Christensen L, Sernert N, Read J, Perko M. Long-term clinical and ultrasound evaluation after arthroscopic acromioplasty in patients with partial rotator cuff tears. Arthroscopy 2006;22(1):44-9. doi: 10.1016/j.arthro.2005.07.027.

27. Keener JD, Galatz LM, Teefey SA, Middleton WD, Steger-May K, Stobbs-Cucchi G, et al. A prospective evaluation of survivorship of asymptomatic degenerative rotator cuff tears. J Bone Joint Surg Am 2015;97(2):89-98. doi: 10.2106/jbjs.n.00099. 


\section{2-year follow-up of unrepaired cuff tears}

28. Kirkley A, Alvarez C, Griffin S. The development and evaluation of a disease-specific quality-of-life questionnaire for disorders of the rotator cuff: The Western Ontario Rotator Cuff Index. Clin J Sport Med 2003;13(2):84-92.

29. Kluczynski MA, Isenburg MM, Marzo JM, Bisson LJ. Does Early Versus Delayed Active Range of Motion Affect Rotator Cuff Healing After Surgical Repair? A Systematic Review and Meta-analysis. Am J Sport Med 2015. doi: 10.1177/0363546515582032.

30. Kuhn JE, Dunn WR, Ma B, Wright RW, Jones G, Spencer EE, et al. Interobserver agreement in the classification of rotator cuff tears. Am J Sport Med 2007;35(3):437-41. doi:

10.1177/0363546506298108.

31. Kukkonen J, Joukainen A, Lehtinen J, Mattila KT, Tuominen EK, Kauko T, et al. Treatment of Nontraumatic Rotator Cuff Tears: A Randomized Controlled Trial with Two Years of Clinical and Imaging Follow-up. J Bone Joint Surg Am 2015;97(21):1729-37. doi: 10.2106/jbjs.n.01051.

32. Lafosse L, Brozska R, Toussaint B, Gobezie R. The outcome and structural integrity of arthroscopic rotator cuff repair with use of the double-row suture anchor technique. J Bone Joint Surg Am 2007;89(7):1533-41. doi: 10.2106/jbjs.f.00305.

33. Maman E, Harris C, White L, Tomlinson G, Shashank M, Boynton E. Outcome of nonoperative treatment of symptomatic rotator cuff tears monitored by magnetic resonance imaging. J Bone Joint Surg Am 2009;91(8):1898-906. doi: 10.2106/jbjs.g.01335.

34. Moosmayer S, Heir S, Smith HJ. Sonography of the rotator cuff in painful shoulders performed without knowledge of clinical information: results from 58 sonographic examinations with surgical correlation. J Clin Ultrasound 2007;35(1):20-6. doi: 10.1002/jcu.20286.

35. Moosmayer S, Smith HJ, Tariq R, Larmo A. Prevalence and characteristics of asymptomatic tears of the rotator cuff: an ultrasonographic and clinical study. J Bone Joint Surg Br 2009;91(2):196-200. doi: 10.1302/0301-620x.91b2.21069. 


\section{2-year follow-up of unrepaired cuff tears}

36. Moosmayer S, Tariq R, Stiris M, Smith HJ. The natural history of asymptomatic rotator cuff tears: a three-year follow-up of fifty cases. J Bone Joint Surg Am 2013;95(14):1249-55. doi: 10.2106/jbjs.I.00185.

37. Moosmayer S, Lund G, Seljom US, Haldorsen B, Svege IC, Hennig T, et al. Tendon repair compared with physiotherapy in the treatment of rotator cuff tears: a randomized controlled study in 103 cases with a five-year follow-up. J Bone Joint Surg Am 2014;96(18):1504-14. doi: 10.2106/jbjs.m.01393. 38. Neer CS, 2nd, Craig EV, Fukuda H. Cuff-tear arthropathy. J Bone Joint Surg Am 1983;65(9):123244. doi.

39. Blinded for review purposes.Norlin R, Adolfsson L. Small full-thickness tears do well ten to thirteen years after arthroscopic subacromial decompression. J Shoulder Elbow Surg 2008;17(1 Suppl):12S-6S. doi: 10.1016/j.jse.2007.06.020.

40. Nove-Josserand L, Edwards TB, O'Connor DP, Walch G. The acromiohumeral and coracohumeral intervals are abnormal in rotator cuff tears with muscular fatty degeneration. Clin Orthop Relat Res 2005(433):90-6.

41. Safran O, Schroeder J, Bloom R, Weil Y, Milgrom C. Natural history of nonoperatively treated symptomatic rotator cuff tears in patients 60 years old or younger. Am J Sport Med 2011;39(4):7104. doi: $10.1177 / 0363546510393944$.

42. Saupe N, Pfirrmann CW, Schmid MR, Jost B, Werner CM, Zanetti M. Association between rotator cuff abnormalities and reduced acromiohumeral distance. AJR Am J Roentgenol 2006;187(2):376-82. doi: 10.2214/ajr.05.0435.

43. Scheibel M, Lichtenberg S, Habermeyer P. Reversed arthroscopic subacromial decompression for massive rotator cuff tears. J Shoulder Elbow Surg 2004;13(3):272-8. doi:

$10.1016 / \mathrm{s} 1058274604000242$.

44. Seigel DG, Podgor MJ, Remaley NA. Acceptable values of kappa for comparison of two groups. Am J Epidem 1992;135(5):571-8. 


\section{2-year follow-up of unrepaired cuff tears}

45. Tashjian RZ. Epidemiology, natural history, and indications for treatment of rotator cuff tears. Clin Sport Med 2012;31(4):589-604. doi: 10.1016/j.csm.2012.07.001.

46. Teefey SA, Rubin DA, Middleton WD, Hildebolt CF, Leibold RA, Yamaguchi K. Detection and quantification of rotator cuff tears. Comparison of ultrasonographic, magnetic resonance imaging, and arthroscopic findings in seventy-one consecutive cases. J Bone Joint Surg Am 2004;86-A(4):70816.

47. Thes A, Hardy P, Bak K. Decision-making in massive rotator cuff tear. Knee Surg Sports Traumatol Arthrosc 2015;23(2):449-59. doi: 10.1007/s00167-014-3470-y.

48. Yamamoto A, Takagishi K, Osawa T, Yanagawa T, Nakajima D, Shitara H, et al. Prevalence and risk factors of a rotator cuff tear in the general population. J Should Elbow Surg 2010;19(1):116-20. doi: 10.1016/j.jse.2009.04.006.

49. Zingg PO, Jost B, Sukthankar A, Buhler M, Pfirrmann CW, Gerber C. Clinical and structural outcomes of nonoperative management of massive rotator cuff tears. J Bone Joint Surg Am 2007;89(9):1928-34. doi: 10.2106/jbjs.f.01073.

50. Zvijac JE, Levy HJ, Lemak L. Arthroscopic subacromial decompression in the treatment of full thickness rotator cuff tears: a 3- to 6-year follow-up. Arthroscopy 1994;10(5):518-23. 
22-year follow-up of unrepaired cuff tears

\section{Figures and Table Legends}

Figure 1. Flow-chart of the inclusion process.

Table I. Demographic data, operative findings at index operation and available data at followup.

Table II. Tear progression from index operation to follow-up.

Table III. Radiographic cuff tear arthropathy classification at follow-up in relation to tendon status at index operation.

Table IV: Clinical scores including pain domain of WORC at follow-up in relation to tear type and radiographic outcome at follow-up.

Table V. Reoperations after the index operation according to tendon status at index operation. 


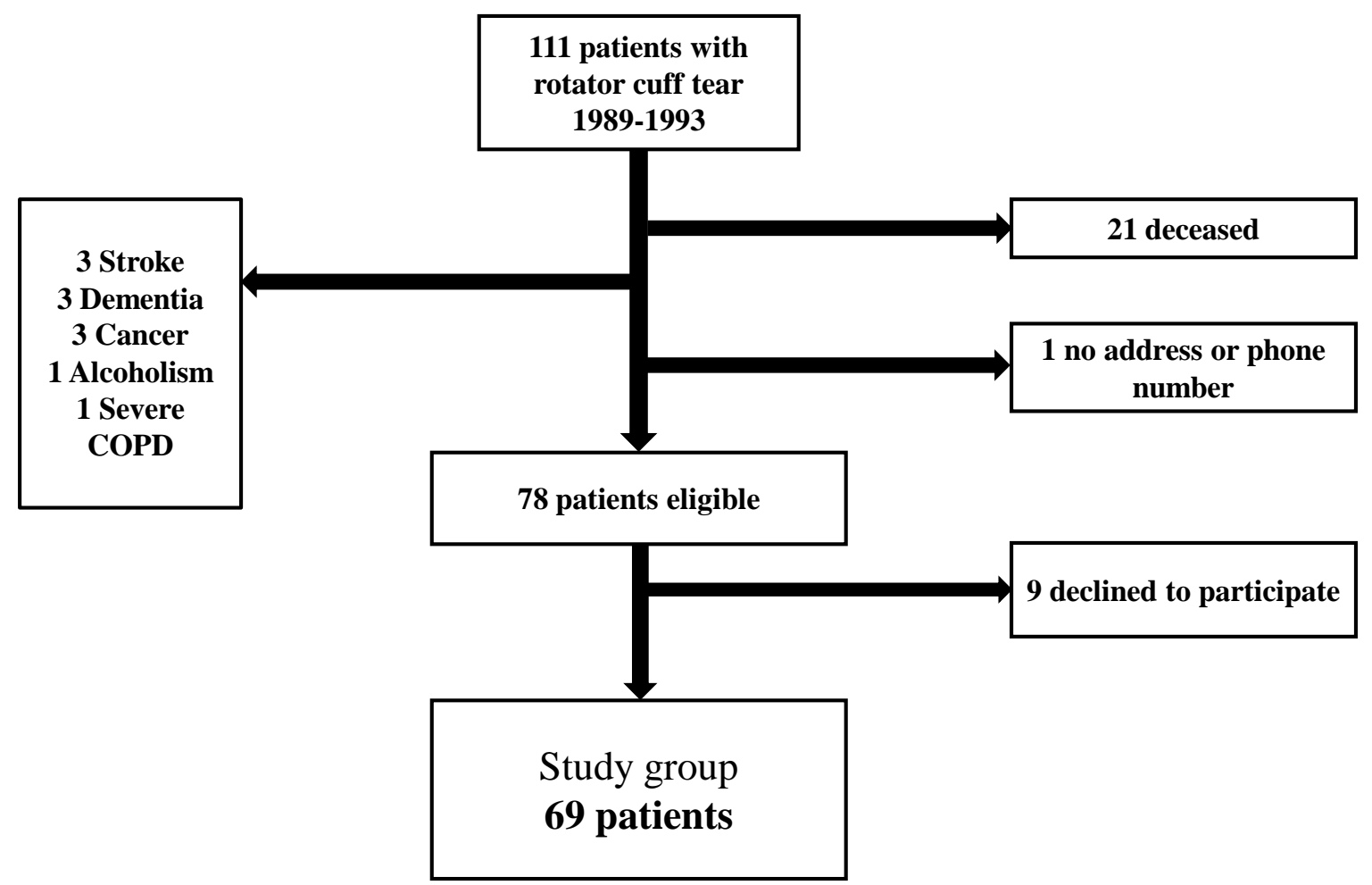

Figure 1. Flow-chart of the inclusion process. 
Table I. Demographic data, operative findings at index operation and available data at follow-up.

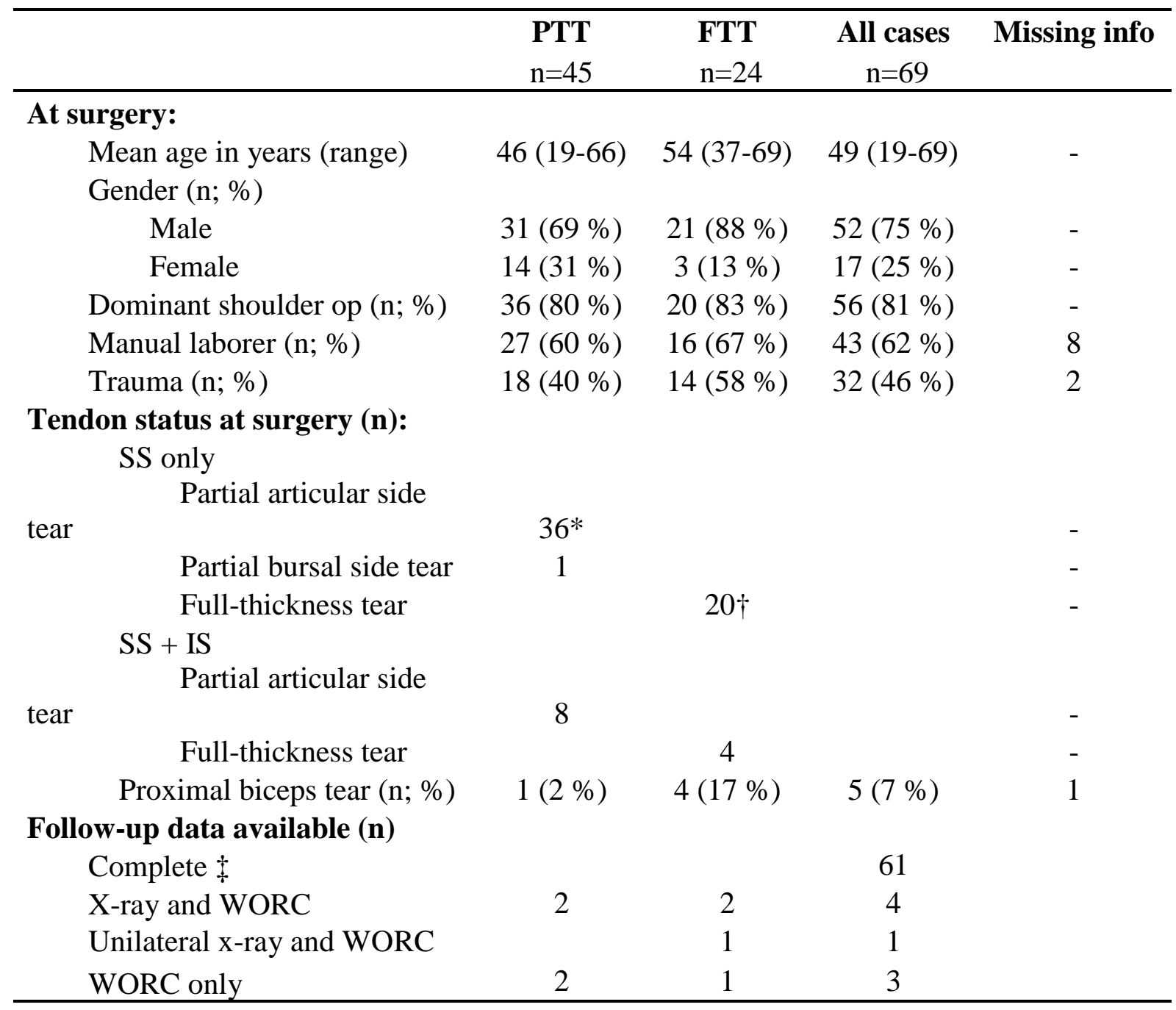

*1 patient also had a partial articular side tear of the subscapularis. $† 3$ patients also had partial articular side tear of subscapularis. $\ddagger 2$ patients lacked only DASH. Abbreviations: FTT, Full-thickness tear; PTT, Partial thickness tear; SS, Supraspinatus; IS, Infraspinatus; US, Ultrasound; CM, Constant-Murley score; WORC, Western Ontario Rotator cuff index; ASD, Arthroscopic subacromial decompression; RC, Rotator Cuff; AC, acromioclavicular joint; Index op, the first acromioplasty. 
Table III. Radiographic cuff tear arthropathy classification at follow-up in relation to tendon status at index operation.

\section{Tendon status at index operation}

Radiographic cuff tear arthropathy

Partial-thickness tear Full-thickness tear p-value

classification

$\mathrm{n}=45$

$\mathrm{n}=24$

Hamada 1

Hamada 2

$40(93 \%)$

$6(26 \%)$

$<0,001$

Hamada 3

$2(5 \%)$

$7(30 \%)$

0,007

Hamada 4 b

Missing x-ray

$1(2 \%)$

2

$3(13 \%)$

0,039

0

7 (30\%)

0,002

Hamada $\geq 2$

$3(7 \%)$

$17(74 \%)$

$<0,001$

Hamada $\geq 3$

$1(2 \%)$

$10(43 \%)$

$<0,001$

() =percentage of patients with available x-ray in each group. Hamada: radiographic cuff tear arthropathy according to Hamada classification ${ }^{19}$. Note: There were no Hamada grade 4 a or 5. 
22-year follow-up of unrepaired cuff tears

Table V. Reoperations after the index operation according to tendon status at index operation.

\begin{tabular}{lccc}
\hline Reoperations (n ; \%) & $\begin{array}{c}\text { PTT } \\
\mathrm{n}=45\end{array}$ & $\begin{array}{c}\text { FTT } \\
\mathrm{n}=24\end{array}$ & $\begin{array}{c}\text { All cases } \\
\mathrm{n}=69\end{array}$ \\
\hline ASD & $5(11 \%)$ & $2(8 \%)$ & $7(10 \%)$ \\
RC-repair & - & $2(8 \%)$ & $2(3 \%)$ \\
Dacron patch & - & $3(13 \%)$ & $3(4 \%)$ \\
AC-resection & $1(2 \%)$ & - & $1(1 \%)$ \\
Diagnostic arthroscopy & $1(2 \%)$ & - & $1(1 \%)$ \\
Open capsular shift & $1(2 \%)$ & - & $1(1 \%)$ \\
Total & $8(18 \%)$ & $7(29 \%)$ & $15(22 \%)$ \\
\hline $\begin{array}{l}\text { () indicates the percentage of patients in each vertical group. Abbreviations: PTT, Partial } \\
\text { thickness tear; FTT, Full-thickness tear; ASD, Arthroscopic subacromial decompression; }\end{array}$ \\
RC-repair, Rotator cuff repair; AC, acromioclavicular joint.
\end{tabular}


Table II. Tear progression from index operation to follow-up.

\begin{tabular}{|c|c|c|c|c|c|c|c|}
\hline \multirow{2}{*}{$\begin{array}{l}\text { Tendon status at index } \\
\text { operation }\end{array}$} & \multirow[b]{2}{*}{$\mathrm{n}$} & \multicolumn{6}{|c|}{ Tendon status at follow-up } \\
\hline & & PTT & FTT SS & FTT SS + IS & FTT SS + IS + SSc & Missing info & Tear progression \\
\hline PTT & 45 & $22(58 \%)$ & $9(24 \%)$ & $6(16 \%)$ & $1(3 \%)$ & 7 & $16(42 \%)$ \\
\hline FTT SS & 20 & 0 & $3(16 \%)$ & $6(32 \%)$ & $10(53 \%)$ & 1 & $16(84 \%)$ \\
\hline FTT SS + IS & 4 & 0 & 0 & 0 & $4(100 \%)$ & - & $4(100 \%)$ \\
\hline Total & 69 & 22 & 12 & 12 & 15 & 8 & $36(59 \%)$ \\
\hline
\end{tabular}

() =percentage of patients with ultrasound examination in each group under "Tendon status at index operation". Abbreviations: PTT,

Partial-thickness tear; FTT, Full-thickness tear; SS, Supraspinatus; IS, Infraspinatus; SSc, Subscapularis 
Table IV: Clinical scores including pain domain of WORC at follow-up in relation to tear type and radiographic outcome at follow-up.

\begin{tabular}{|c|c|c|c|c|c|c|}
\hline \multirow[b]{2}{*}{ Clinical scores } & \multirow{2}{*}{$\begin{array}{c}\begin{array}{c}\text { PTT at } \\
\text { follow-up }\end{array} \\
\text { Hamada } \\
1 \\
\mathrm{n}=21^{*}\end{array}$} & \multicolumn{5}{|c|}{ FTT at follow-up } \\
\hline & & $\begin{array}{c}\text { Hamada } \\
1 \\
\mathrm{n}=18^{*} \\
\end{array}$ & $\begin{array}{c}\text { Hamada } \\
2 \\
\mathrm{n}=9 \\
\end{array}$ & $\begin{array}{c}\text { Hamada } \\
3 \\
n=3 \\
\end{array}$ & $\begin{array}{c}\text { Hamada } \\
4 \mathrm{~b} \\
\mathrm{n}=8 \\
\end{array}$ & $\begin{array}{c}\text { Hamada } \\
\geq 2 \\
n=20\end{array}$ \\
\hline \multicolumn{7}{|l|}{ CM abs } \\
\hline Mean (SD) & $80(17)$ & $65(28)$ & $53(29)$ & $58(13)$ & $36(14)$ & 47 (23) \\
\hline Median (QR) & $83(18)$ & $72(46)$ & $63(52)$ & $62(26)$ & 37 (17) & 42 (39) \\
\hline \multicolumn{7}{|l|}{ CM rel } \\
\hline Mean (SD) & $101(22)$ & $88(38)$ & $70(34)$ & $71(13)$ & $51(20)$ & $62(27)$ \\
\hline Median (QR) & $103(24)$ & $102(72)$ & $84(56)$ & $62(26)$ & $47(21)$ & $57(49)$ \\
\hline \multicolumn{7}{|l|}{ WORC (\%) } \\
\hline Mean (SD) & $81(20)$ & 72 (26) & $65(32)$ & 62 (19) & 48 (19) & $58(26)$ \\
\hline Median (QR) & $88(20)$ & 79 (47) & $72(34)$ & $71(36)$ & $45(35)$ & 63 (39) \\
\hline \multicolumn{7}{|l|}{ WORC-pain $\dagger$} \\
\hline Question nr. 1 & $13(18)$ & $18(26)$ & 32 (39) & $41(45)$ & 31 (39) & 33 (38) \\
\hline Question nr. 2 & $12(17)$ & $15(25)$ & 27 (35) & $28(38)$ & 25 (29) & $26(31)$ \\
\hline \multicolumn{7}{|l|}{ Question nr. $1 \ddagger \S$} \\
\hline No pain & 11 (52\%) & $9(50 \%)$ & $4(44 \%)$ & 1 (33\%) & $4(50 \%)$ & $9(45 \%)$ \\
\hline Any pain & $10(48 \%)$ & $9(50 \%)$ & $5(56 \%)$ & $2(67 \%)$ & $4(50 \%)$ & $11(55 \%)$ \\
\hline Mild pain & 7 (33\%) & 7 (39\%) & $2(22 \%)$ & $1(33 \%)$ & $1(13 \%)$ & $4(20 \%)$ \\
\hline Moderate pain & $3(14 \%)$ & $1(6 \%)$ & $1(11 \%)$ & - & $1(13 \%)$ & $2(10 \%)$ \\
\hline Severe pain & - & $1(6 \%)$ & $2(22 \%)$ & 1 (33\%) & $2(25 \%)$ & $5(25 \%)$ \\
\hline \multicolumn{7}{|l|}{ Question nr. $2 \ddagger \S$} \\
\hline No pain & 12 (57\%) & $10(56 \%)$ & $4(44 \%)$ & $1(33 \%)$ & $3(38 \%)$ & $8(40 \%)$ \\
\hline Any pain & $9(43 \%)$ & $8(44 \%)$ & $5(56 \%)$ & $2(67 \%)$ & $5(63 \%)$ & $12(60 \%)$ \\
\hline Mild pain & 7 (33\%) & $6(33 \%)$ & $2(22 \%)$ & 1 (33\%) & $3(38 \%)$ & $6(30 \%)$ \\
\hline Moderate pain & $2(10 \%)$ & $1(6 \%)$ & $2(22 \%)$ & 1 (33\%) & $2(25 \%)$ & $5(25 \%)$ \\
\hline Severe pain & - & $1(6 \%)$ & $1(11 \%)$ & - & - & $1(5 \%)$ \\
\hline
\end{tabular}

Note: WORC question nr. 1: "How much sharp pain do you experience in your shoulder (in the last week)?". WORC question nr. 2: "How much constant nagging pain do you experience in your shoulder (in the last week)?".*1 patient with primary osteoarthritis are not included.† mean VAS 0-100 mm (Standard deviation). $\$$ Number of patients (percentage per column).§ Pain scores divided according to Jensen $^{23}$ : no pain=0-4 mm VAS, mild pain 5-44 mm, moderate pain 45-74 mm, severe pain 75-100 mm. Abbreviations: SD, Standard deviation; QR, quartile range; FTT, Full-thickness tear; PTT, Partialthickness tear; Hamada, radiographic cuff tear arthropathy according to Hamada classification 1-5 ${ }^{19}$; CM abs, absolute Constant score; CM rel, age- and gender adjusted Constant-Murley score; VAS, Visual Analog Scale. 